\title{
ON STOCHASTIC DIFFERENTIALS IN HILBERT SPACES
}

\author{
E. M. CABAÑA
}

The stochastic integral of a process with values in a separable Hilbert space with respect to Brownian operators has been defined in [1], and it was proved that the corresponding stochastic differential equations have unique solutions under natural assumptions.

We shall give here a rather different definition of Brownian operator; the stochastic integrals with respect to these new Brownian operators may be defined in the same way, and stochastic differential equations may be considered as before. The principal result is that the Levy-I tô formula for stochastic differentiation of composite functions may be extended to the present case.

The suggestions and comments made to the author by Professor H. P. McKean are gratefully acknowledged.

1. Brownian operators. Let $(\Omega, Q, P)$ be a probability space, and let $H$ be a separable real Hilbert space. Since $H$ is separable, a function $x: \Omega \rightarrow H$ is strongly measurable if and only if it is weakly measurable. The measurable functions $x: \Omega \rightarrow H$ will be called $H$-valued random variables. We denote by $H_{P}$ the Hilbert space of $H$-valued random variables for which the norm $\|x\|_{P}^{2}=\left(E\left\{\|x\|^{2}\right\}\right)^{1 / 2}$ is finite, and denote the inner product by $(\cdot, \cdot)_{P}$.

Let $J$ be the interval $[0, T)$ and let $\mu$ be Lebesgue measure on $J$ with the $\sigma$-field $\mathcal{F}$ of Borel sets. If, in the previous definition of $H_{P}$, we replace $(\Omega, Q, P)$ by $(J, F, \mu)$, the Hilbert space thus obtained will be denoted by $H_{\mu}$. We shall adjoin to any symbol denoting a Hilbert space the indexes $P$ or $\mu$ in order to denote the corresponding spaces constructed as above; the corresponding norms and inner products will be denoted by the same indexes. For instance, $\|x\|_{P^{\mu}}^{2}$ $=\int_{J} E\left\{\|x(t, \cdot)\|^{2}\right\} d t$.

The symbol $\Delta$ with or without indexes will stand for an interval of $J$ of the form $\Delta=[r, s)$; its endpoints will be denoted by $\Delta^{-}=r$ and $\Delta^{+}=s$, and the inequalities $\Delta \leqq t, \Delta^{\prime}<\Delta$ mean that no point in $\Delta$ is larger than $t$, and no point in $\Delta^{\prime}$ is larger than any in $\Delta$. Given any process $\zeta$ with domain $J$, its increment $\zeta\left(\Delta^{+}\right)-\zeta\left(\Delta^{-}\right)$will be denoted by $\zeta(\Delta)$.

Let us introduce now the definition of Brownian operator; in what follows, $H$ and $K$ are two fixed separable Hilbert spaces.

Received by the editors July $12,1967$. 
Definition 1.1. A Brownian operator on $H$ to $K$ is the pair $\bar{\beta}=(B, \beta)$ where $B$ is a decreasing family of subfields of $a: B(t)(t \in J)$, and $\beta$ is a mapping from $J$ into bounded linear operators from $H$ to $K_{P}$ such that

$\left(b_{0}\right)$ the random variables in the range of $\beta(\Delta)$ are $B\left(\Delta^{-}\right)$-measurable,

$\left(b_{1}\right)$ for any $t \in J$, the random variables in the range of $\{\beta(\Delta) \mid \Delta \leqq t\}$ are independent of $B(t)$,

$\left(b_{2}\right)$ the processes $\{(\beta h, k) \mid h \in H, k \in K\}$ are a family of scalar Brownian motions, that is, every finite linear combination of the processes of the family is a new real Brownian motion up to a constant factor,

$\left(b_{3}\right)$ for every $h \in H$ and every $\Delta \subset J,\|\beta(\Delta) h\|_{P}^{2}=\|h\|^{2} \mu(\Delta)$, and there exists a constant $q$ such that $E\left\{\|\beta(\Delta) h\|^{4}\right\} \leqq q\|h\|^{4} \mu^{2}(\Delta)$.

Given a Brownian operator $\bar{\beta}=(B, \beta)$, let $H_{P}(\bar{\beta}, t)$ be the subspace of $H_{P}$ generated by the stochastic variables $x \in H_{P}$ independent of $B(t)$. Then the operator $\beta(\Delta): H \rightarrow K_{P}$ may be extended to $H_{P}\left(\bar{\beta}, \Delta^{-}\right)$ in the following way. Let $h_{i} \in H, \Omega_{i} \in \mathbb{B}\left(\Delta^{-}\right)(i=1,2, \cdots, n)$ be given; then we define $\beta(\Delta) \sum_{i} \chi_{\Omega_{i}} h_{i}=\sum_{i} \chi_{\Omega_{i}} \beta(\Delta) h_{i}$, and it follows that $\left\|\beta(\Delta) \sum_{i} \chi_{\Omega_{i}} h_{i}\right\|_{P}^{2}=\left\|\sum_{i} \chi_{\Omega_{i}} h_{i}\right\|_{P}^{2} \mu(\Delta)$, so that $\beta(\Delta)$ may be extended to the closure of the set of finite sums of the form $\sum_{i} \chi_{\Omega_{i}} h_{i}$, namely $H_{P}\left(\bar{\beta}, \Delta^{-}\right)$, and furthermore $\|\beta(\Delta) x\|_{P}^{2}=\|x\|_{P}^{2} \mu(\Delta)$ for any $x \in H_{P}\left(\bar{\beta}, \Delta^{-}\right)$.

Lemma 1.1. Given a Brownian operator $\bar{\beta}=(\beta, \beta)$ on $H$ to $K$, two intervals $\Delta<\Delta^{\prime}$ in $J$ and two random variables $x \in H_{P}\left(\bar{\beta}, \Delta^{-}\right), x^{\prime}$ $\in H_{P}\left(\bar{\beta}, \Delta^{\prime}-\right)$, the extensions of $\beta(\Delta), \beta\left(\Delta^{\prime}\right)$ satisfy:

(i) $E\{\beta(\Delta) x\}=0$,

(ii) $\|\beta(\Delta) x\|_{P}^{2}=\|x\|_{P}^{2} \mu(\Delta)$,

(iii) $\left(\beta(\Delta) x, \beta\left(\Delta^{\prime}\right) x^{\prime}\right)_{P}=0$.

Part $\mathrm{i}$ is obvious, part ii has already been proved, and part iii follows from the fact that $\beta\left(\Delta^{\prime}\right)$ is independent of $\beta(\Delta) x$ and of $x^{\prime}$.

Definition 1.2. Given a Brownian operator $\bar{\beta}=(\Theta, \beta)$, the process $\zeta$ with domain $J$ is said to be nonanticipating with respect to $\bar{\beta}$ when for each $t \in J$, the set $\left\{\zeta\left(t^{\prime}\right) \mid t^{\prime} \leqq t\right\}$ is independent of $\leftrightarrow(t)$. (See [5]; this definition corresponds to Itô's property $(\alpha),[3]$.)

Definition 1.3. A process $\zeta$ with domain $J$ is said to be simple when there exists a partition $\odot$ of the interval $J$ such that the restriction of $\zeta$ to each $\Delta \in \mathcal{P}$ is a constant random variable.

The following lemma has been essentially proved in [1, Lemmas $3,4.3,5.3]$.

Lemma 1.2. Let $\bar{\beta}$ be a Brownian operator on $H$ to $K$. Then the set of simple nonanticipating processes is dense among all nonanticipating processes. 
2. Stochastic integral. Given a simple nonanticipating process $\xi \in H_{P \mu}$ of the form $\sum_{i} \chi_{\Delta_{i}} x_{i}$ with $x_{i}$ independent of $B\left(\Delta_{i}\right)$, define

$$
\int_{J}(d \beta) \xi=\sum \beta\left(\Delta_{i}\right) x_{i}
$$

By Lemma 1.1,

$$
\left\|\int_{J}(d \beta) \xi\right\|_{P}^{2}=\|\xi\|_{P_{\mu}}^{2}
$$

and taking limits in the obvious manner, we may define the stochastic integral $\int_{J}(d \beta) \xi$ for any nonanticipating $\xi \in H_{P_{\mu}}$ so that (1) still holds. Moreover, it is obvious that $E\left\{\int_{J}(d \beta) \xi\right\}=0$.

THEOREM 2.1. Given a nonanticipating $\xi \in H_{P_{\mu}}$ and the Brownian operator $\bar{\beta}$ on $H$ to $K$, the stochastic integral $\int_{0} d \beta(t) \xi(t)$ has a continuous version.

Itô's proof of the existence of continuous versions in the onedimensional case [2, Theorem 8] is based in the validity of the conclusion for the integrals of simple processes, and may be applied here; the generalization of Kolmogorov's inequality used to pass from simple to general $\xi$ is also valid in the infinite-dimensional case, as has been shown in [1, Lemma 5.1]. It remains to show the existence of continuous versions in the case in which $\xi$ is simple, and there is no loss in assuming that $\xi$ is constant as a function of $t$, and bounded'as a function of $\omega \in \Omega$. Given such a $\xi$, the inequality

$$
E\left\{\left\|\int_{\Delta} d \beta(t) \xi\right\|^{4}\right\}=E\left\{\|\beta(\Delta) \xi\|^{4}\right\} \leqq q E\left\{\|\xi\|^{4}\right\} \mu^{2}(\Delta)
$$

holds, since the fact that $\xi$ is nonanticipating means that it is independent of $\bar{\beta}$. This permits us to apply a theorem of Kolmogorov [6] which states that if $\alpha>0, \beta>1$ and $\gamma$ is a process with values in a metric space such that for each interval $\Delta$

$$
E\{|\gamma(\Delta)| \alpha\} \leqq C \mu^{\beta}(\Delta)
$$

(where $C$ is a constant and $|\gamma|$ denotes $\operatorname{dist}(\gamma, 0)$ ), then $\gamma$ has a continuous version. Here $\alpha=4, \beta=2$ and $C=q E\{\|\xi\| 4\}$ and the result follows.

3. Stochastic differentiation of composite functions. Let $\phi: K \rightarrow R$ be a function with first and second derivatives $D \phi: K \rightarrow K$ and $D^{2} \phi: K \rightarrow L(K, K)$, such that, for $k_{0}, k \in K, D^{2} \phi\left(k_{0}\right)$ is bounded, symmetric, and continuous in the operator topology, and 


$$
\phi\left(k_{0}+k\right)=\phi\left(k_{0}\right)+\left(D \phi\left(k_{0}\right), k\right)+\frac{1}{2}\left(D^{2} \phi\left(k_{0}\right) k, k\right)+o_{2}(k),
$$

where $\lim _{n_{k} \|_{\rightarrow 0}}\|k\|{ }^{-2} o_{2}(k)=0$.

We shall consider the stochastic integral

$$
\eta(t)=\eta^{0}+\int_{0}^{t} w(\tau) d \tau+\int_{0}^{t} d \beta(\tau) x(\tau)
$$

where $w \in K_{P_{\mu}}$ and $x \in H_{P_{\mu}}$ are nonanticipating processes, and we shall prove that, for $t \geqq 0$,

$$
\begin{aligned}
\phi(\eta(t))= & \phi\left(\eta^{0}\right)+\int_{0}^{t}(D \phi(\eta(\tau)), w(\tau)) d \tau+\int_{0}^{t}(D \phi(\eta(\tau)), d \beta(\tau) x(\tau)) \\
& +\frac{1}{2} \int_{0}^{t}\left(D^{2} \phi(\eta(\tau)) d \beta(\tau) x(\tau), d \beta(\tau) x(\tau)\right),
\end{aligned}
$$

thus generalizing Itô's formula [3].

The meaning of the integrals in (1) is explained in the following.

The first integral requires no explanation. In order to deal with the second, suppose that $x=\sum x_{\Delta} \chi_{\Delta}$ is a simple nonanticipating process in $H_{P_{\mu}}$ and $\psi=\sum \psi_{\Delta} \chi_{\Delta}$ is a $K$-valued simple nonanticipating process such that $\|\psi\|$ is bounded by a constant $C$. We define

$$
\int_{J}(\psi, d \beta x)=\sum_{\Delta}\left(\psi_{\Delta}, \beta(\Delta) x_{\Delta}\right)
$$

and from

$$
E\left\{\left|\int_{J}(\psi, d \beta x)\right|^{2}\right\} \leqq E\left\{\sum_{\Delta}\left\|\psi_{\Delta}\right\|\left\|^{2}\right\| x_{\Delta} \|^{2} \mu(\Delta)\right\},
$$

the inequality

$$
E\left\{\left|\int_{J}(\psi, d \beta x)\right|^{2}\right\} \leqq E\left\{\int_{J}\|\psi(t)\|^{2}\|x(t)\|^{2} d t\right\} \leqq C^{2}\|x\|_{P_{\mu}}^{2}
$$

follows. Then we can extend the definition of $\int_{J}(\psi, d \beta x)$ for nonanticipating $x \in H_{P_{\mu}}$, by taking a sequence $\left(x_{n}\right)$ of simple nonanticipating processes such that $\left\|x_{n}-x\right\|_{P_{\mu} \rightarrow 0}$ and passing to the limit in $\int_{J}\left(\psi, d \beta x_{n}\right)$. Now $E\left\{\left|\int_{J}(\psi, d \beta x)\right|^{2}\right\} \leqq E\left\{\sum_{\Delta}\left\|\psi_{\Delta}\right\|{ }^{2} \int_{\Delta}\|x(t)\|{ }^{2} d t\right\}$ holds and (2) follows again. For any nonanticipating $\psi$ which is a Borel function of $t$ and satisfies $\|\psi\| \leqq C$, and any nonanticipating $x \in H_{P_{\mu}}$, the definition is extended by taking a sequence of simple nonanticipating uniformly bounded processes $\left(\psi_{n}\right)$, such that ess $\sup _{t \in J}\left\|\psi_{n}(t)-\psi(t)\right\| \rightarrow 0$ as $n \rightarrow \infty$, and setting 


$$
\int_{J}(\psi, d \beta x)=\lim _{n \rightarrow \infty} \int_{J}\left(\psi_{n}, d \beta x\right)
$$

This integral has a continuous version in $t$, and satisfies (2). Now it can be further extended to the case in which $P\left\{{\left.\operatorname{ess~} \sup _{t \in J}\|\psi(t)\|<\infty\right\}}\right.$ $=1$, and this is the case of the second integral of (1) with $D \phi$ instead of $\psi$, since $\eta$ and hence $D \phi(\eta)$ is a.s. continuous.

As to the third integral, consider a nonanticipating process $x \in H_{P \mu}$ and a bounded symmetric operator-valued nonanticipating process $\psi$ with $\|\psi\| \leqq C<\infty$. Given any interval $\Delta C J$, let us define

$$
\left\langle\psi\left(\Delta^{-}\right) \beta(\Delta) x\left(\Delta^{-}\right), \beta(\Delta) x\left(\Delta^{-}\right)\right\rangle
$$

as the mean over $\beta(\Delta)$ of $\left(\psi\left(\Delta^{-}\right) \beta(\Delta) x\left(\Delta^{-}\right), \beta(\Delta) x\left(\Delta^{-}\right)\right)$. For simple $\psi=\sum_{\Delta} \psi_{\Delta} \chi_{\Delta}$ and $x=\sum_{\Delta} x_{\Delta} \chi_{\Delta}$, we define

$$
\int_{J}(\psi d \beta x, d \beta x)=\sum_{\Delta}\left\langle\psi_{\Delta} \beta(\Delta) x_{\Delta}, \beta(\Delta) x_{\Delta}\right\rangle
$$

The estimate

$$
\left|\left\langle\psi_{\Delta} \beta(\Delta) x_{\Delta}, \beta(\Delta) x_{\Delta}\right\rangle\right| \leqq\left\|\psi_{\Delta}\right\|\left\|x_{\Delta}\right\|^{2} \mu(\Delta)
$$

leads to

$$
E\left\{\left|\int_{J}(\psi d \beta x, d \beta x)\right|\right\} \leqq E\left\{\int_{J}\|\psi(t)\|\|x(t)\|^{2} d t\right\}
$$

therefore, using now convergence in the mean, the definition (3) is extended as above for nonanticipating $x \in H_{P_{\mu}}$ and processes $\psi$ which are Borel functions of $t$, such that $\|\psi\| \leqq C$; the integral has a continuous version in $t$, and satisfies (4). On the other hand, the estimate

$$
\begin{aligned}
& E\left\{\mid \sum_{\Delta}\left(\left(\psi\left(\Delta^{-}\right) \beta(\Delta) x\left(\Delta^{-}\right), \beta(\Delta) x\left(\Delta^{-}\right)\right)\right.\right. \\
&\left.\left.-\left\langle\psi\left(\Delta^{-}\right) \beta(\Delta) x\left(\Delta^{-}\right), \beta(\Delta) x\left(\Delta^{-}\right)\right\rangle\right)\left.\right|^{2}\right\} \leqq \text { constant } \times \sum_{\Delta} \mu^{2}(\Delta)
\end{aligned}
$$

shows that if $\max _{\Delta} \mu(\Delta) \rightarrow 0$, then

$$
\begin{aligned}
E\left\{\mid \sum_{\Delta}\left(\psi\left(\Delta^{-}\right) \beta(\Delta) x\left(\Delta^{-}\right), \beta(\Delta) x\left(\Delta^{-}\right)\right)\right. \\
\\
\left.\quad-\sum_{\Delta}\left\langle\psi\left(\Delta^{-}\right) \beta(\Delta) x\left(\Delta^{-}\right), \beta(\Delta) x\left(\Delta^{-}\right)\right\rangle \mid\right\} \rightarrow 0
\end{aligned}
$$


and this justifies the use of the symbol $\int_{J}(\psi d \beta x, d \beta x)$ for the integral just defined. As in the previous case, the integral can further be extended to the case in which $P\left\{\sup _{t \in J}\|\psi(t)\|<\infty\right\}=1$, and this is the case of the third integral of (1), with $D^{2} \phi$ in the place of $\psi$.

Now we proceed to prove (1), noticing first that several reductions can be made. Let us introduce the stopping time

$$
T_{n}=\max \left\{t \in J \mid \text { for } 0 \leqq \tau<t,\|D \phi(\eta(\tau))\|+\left\|D^{2} \phi(\eta(\tau))\right\|<n\right\},
$$

which satisfies $P\left\{\lim _{n \rightarrow \infty} T_{n}=T\right\}=1$, and prove (1) for each stopped process

$$
\eta_{n}(t)=\eta^{0}+\int_{0}^{t} \chi_{\left(0, T_{n}\right)}(\tau) w(\tau) d \tau+\int_{0}^{t} d \beta(\tau) \chi_{\left(0, T_{n}\right)}(\tau) x(\tau) .
$$

This allows us to assume that $D \phi$ and $D^{2} \phi$ are uniformly bounded. Since the integrals in (1) have continuous versions, it is enough to prove the equality for fixed $t$. The process of construction of the integrals shows that it is enough to prove it for simple $w$ and $x$, and hence for constant $w$ and $x$ because of the additivity in $t$. Moreover, the notations will be shortened by setting $w=0$; the proof can be repeated in the same way, taking account of a possibly nonvanishing $w$, with no additional trouble. Furthermore, the constant nonanticipating random variable $x$ must be independent of all the increments $\beta(\Delta)$; hence there is no loss in assuming $x=h \in H$. Finally, with $\beta(0)=0$ and $t=T, \eta(t)$ becomes $\beta(t) h$ and (1) reduces to

(6) $\phi \circ \eta(J)=\int_{J}(D \phi(\eta(\tau)), d \beta(\tau) h)+\frac{1}{2} \int_{J}\left(D^{2} \phi(\eta(\tau)) d \beta(\tau) h, d \beta(\tau) h\right)$ which we prove as follows.

Given a sequence $\left(\mathcal{P}_{n}\right)$ of partitions of $J$ with norm tending to zero, we may write

$$
\begin{array}{r}
\phi \circ \eta(J)=\sum_{\Delta \in \mathbb{P}_{n}}\left[\left(D \phi \circ \eta\left(\Delta^{-}\right), \beta(\Delta) h\right)+\frac{1}{2}\left(D^{2} \phi \circ \eta\left(\Delta^{-}\right) \beta(\Delta) h, \beta(\Delta) h\right)\right. \\
\left.+o_{2}(\beta(\Delta) h)\right] .
\end{array}
$$

The steps in the definitions of $\int_{J}(D \phi(\eta), d \beta h)$ and $\int_{J}\left(D^{2} \phi(\eta) d \beta h, d \beta h\right)$, and the remark (5), show that

(7) $E\left\{\left|\sum_{\Delta \in \mathbb{P}_{n}}\left(D \phi \circ \eta\left(\Delta^{-}\right), \beta(\Delta) h\right)-\int_{J}(D \phi(\eta), d \beta h)\right|^{2}\right\} \rightarrow 0$,

and

$$
n \rightarrow \infty,
$$


(8) $E\left\{\left|\sum_{\Delta \in \mathcal{P}_{n}}\left(D^{2} \phi \circ \eta\left(\Delta^{-}\right) \beta(\Delta) h, \beta(\Delta) h\right)-\int_{J}\left(D^{2} \phi(\eta) d \beta h, d \beta h\right)\right|\right\} \rightarrow 0$, $n \rightarrow \infty$.

Moreover,

$$
\begin{aligned}
& E\left\{\left|\sum_{\Delta \in \mathscr{P}_{n}}\|\beta(\Delta) h\|^{2}-\|h\|^{2} \mu(\Delta)\right|^{2}\right\} \\
= & E\left\{\sum_{\Delta \in \mathbb{P}_{n}}\left(\|\beta(\Delta) h\|^{2}-\|h\|^{2} \mu(\Delta)\right)^{2}\right\} \rightarrow 0
\end{aligned}
$$

as $n \rightarrow \infty$. Therefore a subsequence $\left(\rho_{n_{i}}\right)$ may be chosen in such a way that

$$
\lim \sum_{\Delta \in \mathcal{P}_{n_{i}}} o_{2}(\beta(\Delta) h)=0 \text { a.s. }
$$

Taking account of (7) and (8), it follows that $\left(\rho_{n_{i}}\right)$ may be also chosen in such a way that

$$
\lim \sum_{\Delta \in \mathbb{P}_{n i}}\left(D \phi \circ \eta\left(\Delta^{-}\right), \beta(\Delta) h\right)=\int_{J}(D \phi(\eta), d \beta h) \text { a.s. }
$$

and

$$
\lim \sum_{\Delta \in \mathbb{P}_{n_{i}}}\left(D^{2} \phi \circ \eta\left(\Delta^{-}\right) \beta(\Delta) h, \beta(\Delta) h\right)=\int_{J}\left(D^{2} \phi(\eta) d \beta h, d \beta h\right) \text { a.s. }
$$

and this proves (6).

\section{REFERENCES}

1. E. Cabaña, Stochastic integration in separable Hilbert spaces, Montevideo Publ. Inst. Mat. Estadíst. 4 (1966), 49-80.

2. K. Itô, On stochastic differential equations, Mem. Amer. Math. Soc. No. 4 (1951).

3. - On a formula concerning stochastic differentials, Nagoya Math. J. 3 (1951), 55-65.

4. P. Levy, Processus stochastiques et mouvement brownien, Gauthier-Villars, Paris, 1948.

5. H. P. McKean, Stochastic integrals, Academic Press, New York, (to appear).

6. E. Slutsky, Qualche proposizione relativa alla teoria delle funzioni aleatorie, Giorn. Ist. Ital. Attuari. 8 (1937), 183-199.

THE ROCKEFELLER UNIVERSITY 